\title{
BMJ Open Beliefs about medicines and non- adherence in patients with stroke, diabetes mellitus and rheumatoid arthritis: a cross-sectional study in China
}

Li Wei, ${ }^{1}$ Sarah Champman, ${ }^{1,2}$ Xiaomei Li, ${ }^{3}$ Xin Li, ${ }^{4}$ Sumei Li, ${ }^{5}$ Ruoling Chen, ${ }^{6}$ Nie Bo, ${ }^{1}$ Angel Chater, ${ }^{1,7}$ Robert Horne ${ }^{1}$

To cite: Wei L, Champman S, Li X, et al. Beliefs about medicines and non-adherence in patients with stroke, diabetes mellitus and rheumatoid arthritis: a cross-sectional study in China. BMJ Open 2017;7:e017293. doi:10.1136/ bmjopen-2017-017293

- Prepublication history for this paper is available online. To view these files please visit the journal online (http://dx.doi. org/10.1136/bmjopen-2017017293).

Received 12 April 2017 Revised 12 July 2017 Accepted 28 July 2017

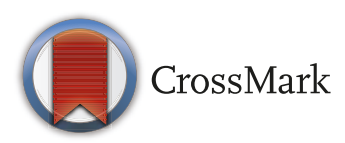

For numbered affiliations see end of article.

Correspondence to Dr LiWei; I.wei@ucl.ac.uk

\section{ABSTRACT}

Objectives To investigate beliefs about medicines and their association with medicine adherence in patients with chronic diseases in China.

Design A cross-sectional questionnaire-based study Setting Two large urban hospitals in Hefei and Tianjin, China

Participants Hospital inpatients (313 stroke patients) and outpatients (315 diabetic patients and 339 rheumatoid arthritis (RA) patients) were recruited between January 2014 and September 2014.

Outcome measures The Beliefs about Medicines Questionnaire (BMQ), assessing patients' beliefs about the specific medicine (Specific-Necessity and SpecificConcerns) prescribed for their conditions (stroke/ diabetes/RA) and more general background beliefs about pharmaceuticals as a class of treatment (BMQ-General Benefit, Harm and Overuse); the Perceived Sensitivity to Medicines scale (PSM) assessed patients' beliefs about how sensitive they were to the effects of medicines and the Medication Adherence Report Scale. The association between non-adherence and beliefs about medicines was assessed using a logistic regression model.

Results Patients with diabetes mellitus had a stronger perceived need for treatment (mean (SD) SpecificNecessity score, $3.75(0.40)$ ) than patients with stroke (3.69 (0.53)) and RA (3.66 (0.44)) $(\mathrm{p}=0.049)$. Moderate correlations were observed between Specific-Concerns and General-Overuse, General-Harm and PSM (Pearson correlation coefficients, $0.39,0.49$ and 0.49 , respectively, $\mathrm{p}<0.01)$. Three hundred and eleven patients were nonadherent to their medicine (159 (51.0\%) in the stroke group, $60(26.7 \%)$ in the diabetes mellitus group and $62(19.8 \%)$ in the RA group, $p<0.01)$. Across the whole sample, after adjusting for demographic characteristics, non-adherence was associated with patients who had higher concerns about their medicines $(0 \mathrm{R}, 1.35,95 \% \mathrm{Cl}$ 1.07 to 1.71) and patients who believed that they were personally sensitive to the effects of medications (OR 1.44, $95 \% \mathrm{Cl} 1.16$ to 1.85$)$.

Conclusion The BMQ is a useful tool to identify patients at risk of non-adherence. In the future, adherence intervention studies may use the BMQ to screen for
Strengths and limitations of this study

- First large study of beliefs about medicines in China.

- High response rate, reflecting generalisability of the study finding.

- A cross-sectional design implicating that the association between non-adherence and beliefs about medicines did not necessarily lead a causal relationship.

- Self-reported adherence may not be the best measurement for medicine adherence.

patients who are at risk of non-adherence and to map interventional support.

\section{INTRODUCTION}

Medicine plays an essential role in chronic disease management. However, it is recognised that only half of patients with chronic diseases take their medicines as prescribed. ${ }^{1}$ Stroke, diabetes mellitus and rheumatoid arthritis (RA) are three common chronic diseases which together affect over $10 \%$ of China's population of 1.40 billion. $^{2-5}$ Stroke is the leading cause of adult death and disability in China with an annual mortality rate of approximately 1.6 million, or 157 per $100000 .^{6}$ Patients with chronic diseases often require multiple medicine treatments for their conditions and any associated symptoms and comorbidities. To obtain full benefit from treatment, patients need to adhere to these complex medicine regimens in order to control their disease and maintain health. However, in reality, medicine management is suboptimal and relates to physician inertia and patients' poor adherence with therapy. ${ }^{7}$ The elderly, with high rates of comorbidity and coprescribing, tend to have poor 
adherence and are at particular risk of unwanted adverse effects from drugs. ${ }^{89}$

Studies outside China have identified patients' beliefs about medicines as an important determinant of non-adherence. ${ }^{10}$ A recent meta-analysis of 96 peer-reviewed studies involving over 24000 patients across 24 longterm conditions and 18 countries showed that non-adherence was related to patients beliefs about medicines, measured by the Beliefs about Medicines Questionnaire (BMQ). ${ }^{10}$ These studies indicated that there was often a disconnect between the patients and prescribers view of the medicine. Many patients doubted their personal need for the treatment or harboured concerns and these beliefs are associated with non-adherence. Beliefs about medicines may also be relevant in China, particularly as there is some evidence that trust between patients and health professionals may have diminished after China's economic reform. ${ }^{11} 12$ Patients often do not trust their doctors and doubt the treatment including therapeutic treatment they received. It is possible that this may translate into scepticism about prescribed medicines and non-adherence. The aim of this study was to understand beliefs about medicines in Chinese patients with stroke, diabetes mellitus and RA and to investigate whether these beliefs are associated with medicine non-adherence.

\section{METHODS}

A cross-sectional study was conducted in two large teaching hospitals in China between January 2014 and November 2014. The study was approved by the local research ethics committees.

\section{Study populations and recruitment}

Patients with stroke were recruited from The Second Hospital, Tianjin Medical University, Tianjin, China and patients with diabetes mellitus and RA were recruited from Anhui Provincial Hospital, Anhui Medical University, Hefei, China. Both hospitals are large teaching hospitals and are level 3 general hospitals, the highest classification for quality care given by China's National Health and Family Planning Commission for all public hospitals. ${ }^{13}$ Patients who had a clinical diagnosis of stroke, diabetes mellitus or RA who were judged by the healthcare professionals as able to answer questions were invited by the healthcare professionals to take part in the study when they were admitted to the hospital (stroke patients) or came to hospital clinics (diabetic and RA patients) during the study period. Some patients may have comorbidity. However, they were only included for the condition they were hospitalised or the condition from the specific outpatient clinics. They were asked to complete a questionnaire which took approximately $10-15 \mathrm{~min}$. Study information was given to patients and verbal consent was obtained before they started to fill in the questionnaire. All invited patients returned their questionnaires. Since there is a lack of research on beliefs about medicines and medicine non-adherence in China, a target sample size of 300 patients for each condition was chosen based on typical sample sizes used in similar surveys. A recent meta-analysis showed that small-moderate effect of psychological beliefs on medicine adherence was observed in 94 studies with an average sample size of $266 .{ }^{10}$ Data collection started in January 2014 and lasted around 4 months for each condition and was stopped once the target numbers were reached (November 2014).

\section{Questionnaire measurement}

The questionnaire consisted of demographic information, the BMQ questionnaire, ${ }^{14}$ perceived sensitivity to medicines scale (PSM) and medicine adherence report scale (MARS) ${ }^{15}$ All scales were translated and back translated in accordance with the Originator's conditions to create a Chinese version, (BMQ-Chinese $\odot$ R Horne MARS-Chinese () R Horne, PSM-Chinese () R Horne). The BMQ and MARS were translated into Chinese by LW, the principal investigator for this study. They were then sent to the coauthors, XL and XL for comments and further adaptation resulting in a final Chinese version of the BMQ.

Demographic information included name, age, gender, education, occupation and duration of the condition. The BMQ questionnaire has two parts: the BMQ-Specific assessing beliefs about medicine used for a particular condition and the BMQ-General assessing beliefs about medicines in general. The details of the BMQ questionnaire can be found in a previous publication. ${ }^{8}$ In brief, BMQ-Specific comprised two scales: a five-item treatment necessity scale Specific-Necessity and a six-item treatment concern scale Specific-Concern. BMQ-General used three subscales: (1) General-Overuse (four items), (2) General-Harm (four items) and (3) General-Benefit (four items). Perceived Sensitivity to Medicines (PSM) scale contained five items assessing perceptions of personal susceptibility to the effects of medicines. All BMQ items and PSM were scored on a Likert type scale (where $1=$ strongly disagree, 2=disagree, 3=uncertain, $4=$ agree and $5=$ strongly agree). A mean item score was then calculated as the sum of each item score divided by the number of items (eg, mean score of Specific-Necessity $=(\mathrm{N} 1+\mathrm{N} 2+\mathrm{N} 3+\mathrm{N} 4+\mathrm{N} 5) / 5)$. The Cronbach's $\alpha$ s indicated that all scale measures were internally consistent in the study sample ${ }^{16}$ with high value of $\alpha_{\mathrm{psm}}=0.85, \alpha_{\text {con- }}$ cern $=0.75$ and $\alpha_{\text {necessity }}=0.64$, and low values of $\alpha_{\text {overuse }}=0.54$, $\alpha_{\text {harm }}=0.55$ and $\alpha_{\text {benefit }}=0.58$. For BMQ-Specific, patients were only asked for the treatment prescribed for the diagnosed condition in the past 12 months. Medicine adherence was measured for the same period using the MARS scale consisting of five items with 25 score points in total. For example, one item is 'I forget to take them'.

Before the study commenced, 30 people including doctors, nurses, medical students and patients were asked to complete the questionnaire to assess whether the Chinese version of the BMQ, PSM and MARS would be easily understood by Chinese patients. Feedback was wholly positive; however, we recognised that patients were 
likely to be elderly and may have poor literacy. Therefore, arrangements were made to provide help should patients have any difficulty completing the questionnaire. Patients in both centres completed the questionnaire either by themselves or with help from the healthcare professionals in the medical ward or clinic they attended. Healthcare professionals received a briefing from the local investigators on how to complete the questionnaire including instructions on how to explain the meaning of the questionnaire items without influencing patients' responses.

\section{Definition of adherence and non-adherence}

It is commonly accepted that it is not necessary to take $100 \%$ of a prescribed treatment, and $80 \%$ adherence is a commonly used cut-off to define adherence, especially in medicine benefit/safety studies. ${ }^{17}$ While the MARS scale does not allow direct assessment of the percentage of prescribed treatment taken, we used a cut-off of 20 out of 25 on this scale to define adherence, indicating that patients are taking a large proportion of their prescribed doses. Adherence was calculated as sum of the scores from each item divided by the maximum points of 25 using the five-item MARS scale. Non-adherent patients were defined as having a score $<80 \%$ adherence to medicine.

\section{Attitudinal analysis}

Participants were categorised into attitudinal groups (ie, Sceptical, Ambivalent, Indifferent and Accepting groups) based on whether they scored above or below the scale midpoint for BMQ necessity and concerns scales. Non-adherence was investigated between the groups. ${ }^{17}$

\section{Statistical analysis}

Data were presented as mean (SD) for continuous variables and as frequencies $(\%)$ for categorical variables. $\chi^{2}$ and analysis of variance tests were performed to determine significant differences between the three diseases. Correlations were used to examine relationships between BMQ-General and BMQ-Specific subscales. Logistic regression analysis was employed to assess the association between non-adherence and medicine beliefs. Five per cent of patients had at least one missing value in their $\mathrm{BMQ}$ answers and the total missing values were $0.3 \%$ for the whole BMQ items. A sensitivity analysis was done to include all patients with missing data replaced with imputed data in the multivariable analysis concluding all variables listed in table 1 . The multiple imputation analysis included all variables used in the final analysis with 10 imputations by using the Markov Chain Monte Carlo method to produce imputed data. All statistical analyses were carried out using SAS (V.9.4).

\section{RESULTS}

\section{Patient characteristics}

There were 967 patients in the study (313 with stroke, 315 with diabetes mellitus and 339 with RA). Table 1 shows the demographic and clinical information by disease groups. Patients with RA were significantly younger than patients with stroke and diabetes mellitus (mean age 49.7 vs 65.8 and 62.5 , respectively). The RA group was $85 \%$ female while only $44 \%$ of stroke patients and $45 \%$ of diabetes mellitus patients were female. Patients with RA were five times more likely to report having received no formal education than patients with stroke and diabetes mellitus (23.3\% vs $4.8 \%$ and $4.1 \%$, respectively). Over half of patients in the stroke group had less than 1 year disease duration while more than half of the patients in the RA and diabetes mellitus groups had more than 5 years disease duration.

\section{BMQ results}

The results of BMQ subscales are shown in table 2. There were no significant differences in the mean scores of Specific-Concerns and General-Harm between the three groups. However, differences were observed for Specific-Necessity, General-Overuse, General-Benefit and PSM among the three groups. Patients with RA had the highest score of 3.75 (SD 0.40) for Specific-Necessity and lowest scores of 2.95 (SD 0.51) for General-Overuse and 3.55 (SD $0.45)$ for General-Benefit. The mean scores of PSM were 2.89 (SD 0.65), 2.35 (SD 0.64) and 2.70 (SD 0.68). The attitudinal analysis categorised patients into four groups (high/low necessity and high/low concerns) according to the midpoint of the Specific-Necessity Specific-Concerns, scales shows that $45 \%$ of patients were classified as 'Ambivalent', $45 \%$ as 'Accepting', $4 \%$ as 'Sceptical' and $6 \%$ as 'Indifferent' (figure 1).

Patients' general beliefs about medicines were associated with their evaluations of the specific treatments they were taking. Table 3 shows the correlation between BMQ-General and BMQ-Specific. Specific-Necessity was positively associated with General-Benefit (Pearson correlation coefficients $=0.31, \mathrm{p}<0.01$ ). Moderate correlations were observed between Specific-Concerns and General-Overuse, General-Harm and PSM (Pearson correlation coefficients, $0.39,0.49$ and 0.49 , respectively, $\mathrm{p}<0.01)$. General-Overuse was also correlated with General-Harm and PSM (Pearson correlation coefficients, 0.45 and 0.39 , respectively, $\mathrm{p}<0.01)$. The Pearson correlation coefficient was $0.39(\mathrm{p}<0.01)$ for General-Harm and PSM.

\section{Association between beliefs about medicines and non- adherence}

Three hundred and eleven patients were non-adherent to their medicine $(159(51.0 \%)$ in the stroke group, 60 $(26.7 \%)$ in the diabetes mellitus group and $62(19.8 \%)$ in the RA group, $\mathrm{p}<0.01)$. After adjusting for demographic characteristics, Specific-Concerns and PSM were significantly associated with non-adherence (adjusted ORs, $1.35,95 \%$ CI 1.07 to 1.71 and $1.44,95 \%$ CI 1.16 to 1.80). Negative associations between non-adherence and Specific-Necessity and General-Benefit were not statistically significant (table 4). The subgroup analyses showed that the point estimates of Specific-Concerns were positively associated with non-adherence across the 
Open Access

Table 1 Patient characteristics by conditions

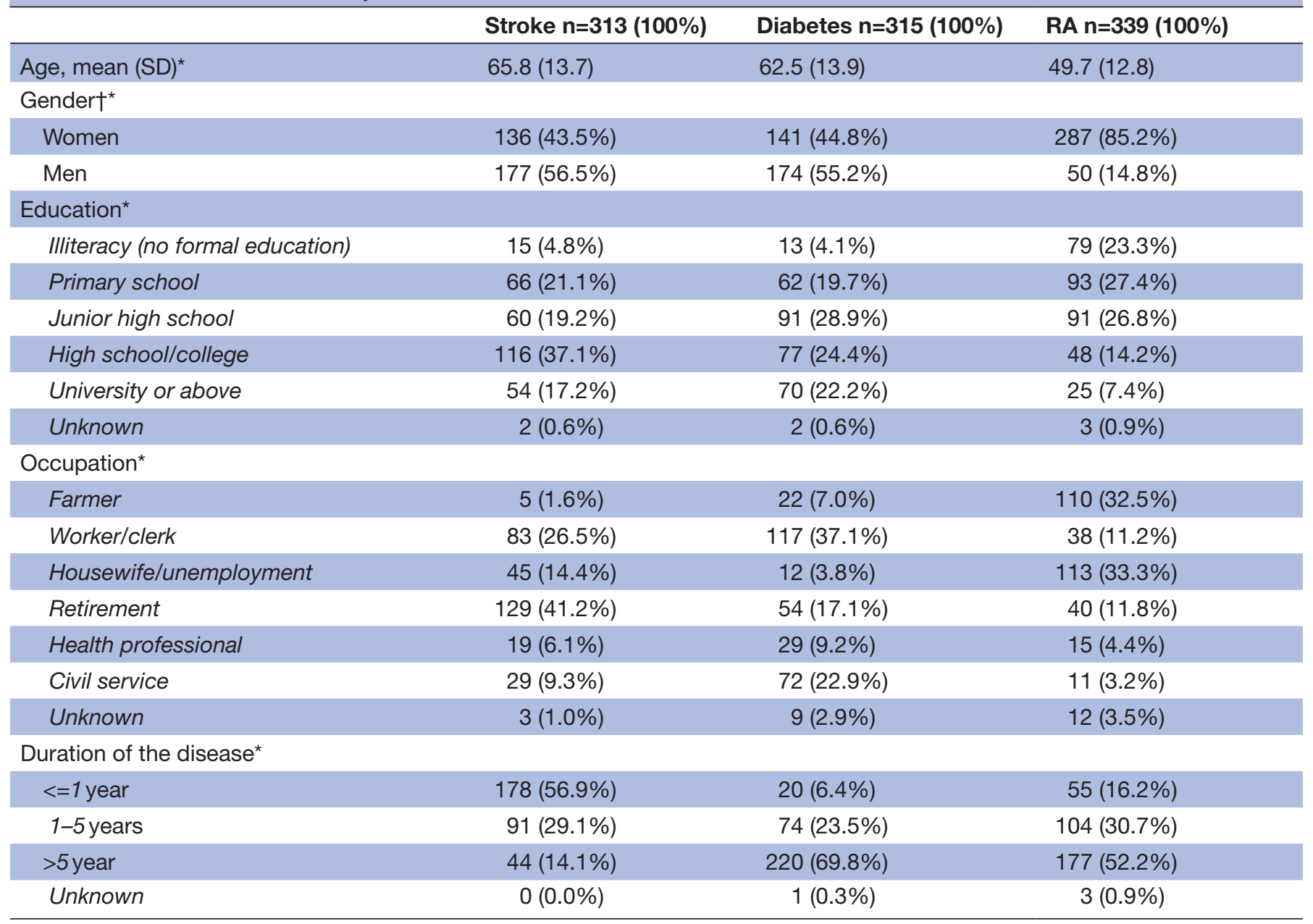

* $<0.05$

†Excluding missing data.

RA, rheumatoid arthritis.

three conditions, that is, the more concerns about the medicines, the more non-adherent to medicines, with adjusted ORs ranging from 1.15 (95\% CI 0.67 to 1.98) for diabetes mellitus to 1.43 (95\% CI 1.02 to 2.00$)$ for stroke (table 5). The unadjusted results showed similar results across the three disease conditions. The sensitivity analysis showed that Specific-Concerns and PSM were positively associated with non-adherence (adjusted ORs, $1.1595 \%$ CI 1.04 to 1.26 and $1.40,95 \%$ CI 1.04 to 1.26 , respectively) and General-Benefit was negatively associated with non-adherence (adjusted OR 0.79, 95\% CI 0.71 to 0.87 ). Compared with patients in the Accepting group, patients in the other attitudinal groups were more likely to be the non-adherent (adjusted ORs, $1.5095 \%$ CI 1.10

Table 2 Results of BMQ and PSM by disease conditions

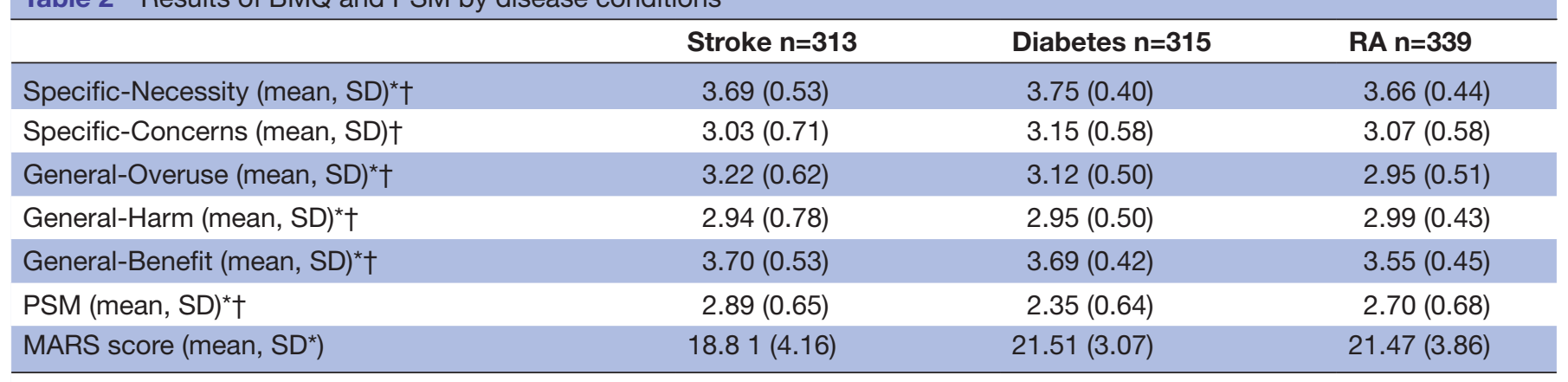

${ }^{*}<0.05$

†Excluding missing data.

MARS, medicine adherence report scale; PSM, perceived sensitivity to medicines; RA, rheumatoid arthritis. 
High Concerns

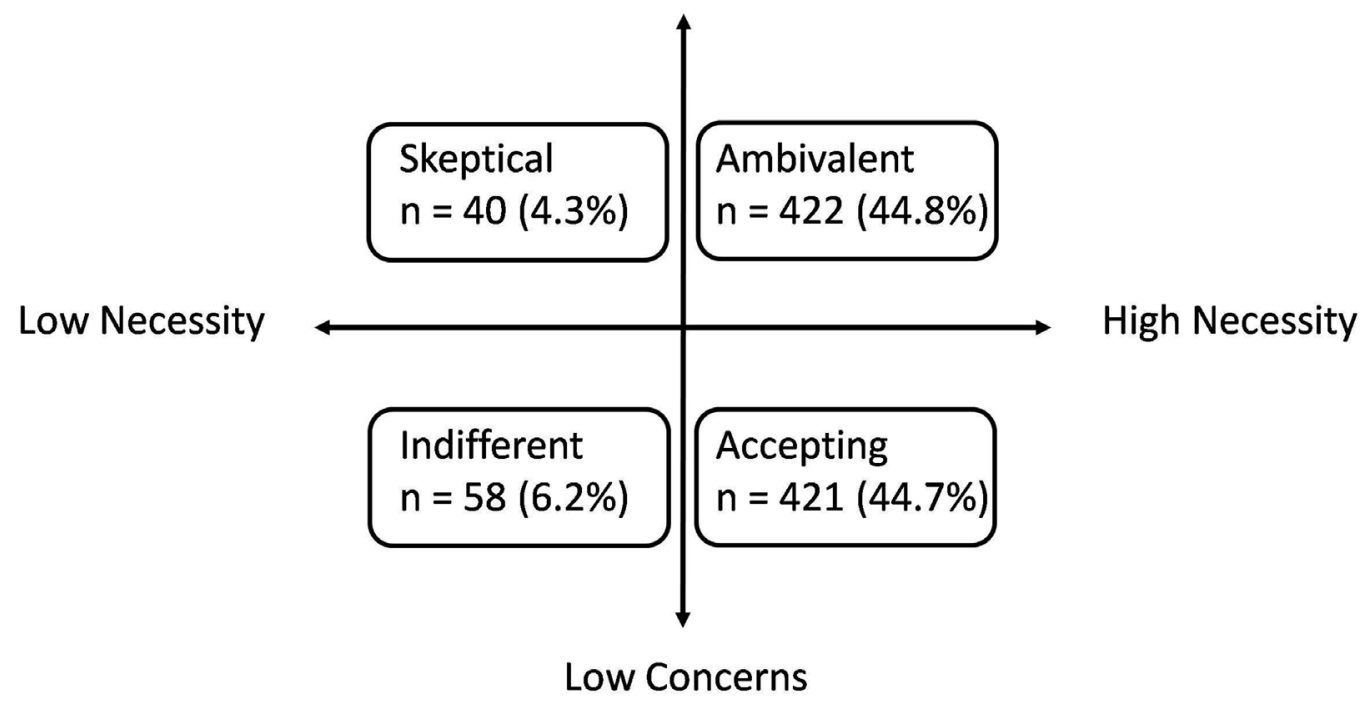

Figure 1 Attitudinal analysis of Beliefs about Medicines Questionnaire (BMQ)-Specific.

to 2.05 for the Ambivalent group, $1.2795 \%$ CI 0.60 to 2.68 for the Sceptical group and 1.27 95\% CI 0.69 to 2.34 for the Indifferent group. Only patients in the Ambivalent group showed a statistical significance.

\section{DISCUSSION}

This is the first large study of this kind that investigates beliefs about medicines and medicine non-adherence in China in three common chronic diseases. Both unadjusted and adjusted results revealed that Specific-Concerns and PSM were significantly associated with non-adherence, that is, the higher the concern about the medicine or the higher perceived sensitivity to medicines. Using the continuous MARS-5 scores showed similar relationships to those reported in logistic regression $(\mathrm{r}=-0.09, \mathrm{p}<0.01$ for Specific-Concerns and $\mathrm{r}=-0.09, \mathrm{p}<0.01$ for PSM). We noted differences between groups that might indicate different attitudes towards the diseases and their treatments or be attributable to other factors. The hospital out-patients (diabetes mellitus and RA) included some patients who had previous hospitalisations and come back to the hospital for regular monitoring.

Our findings were consistent with the evidence from a recent meta-analysis which showed that higher adherence was associated with fewer concerns about treatment ${ }^{10}$ but not consistent with previous findings that higher belief in personal need for treatment was associated with higher adherence; Specific-Necessity was not associated with non-adherence in our overall analysis. Subgroup analyses showed that patients with stroke and diabetes mellitus showed the same direction association as previous studies, that is, patients with higher necessity were less likely to be non-adherent. However, an opposite non-statistically significant result of Specific-Necessity and adherence for patients with RA (Our result of 1.34 (95\% CI 0.73 to 2.46 ) for non-adherence equals 0.75 ,
95\% CI 0.41 to 1.38 for adherence) was observed in the study in comparison with the meta-analysis result which had a pooled OR of $3.28,95 \%$ CI 1.11 to 9.71 for adherence. ${ }^{10}$ The point estimate could be due to chance and further studies need to confirm the finding. A recent study found that General-Benefit may be negatively associated with non-adherence among 398 patients with epilepsy from the UK primary care population (adjusted OR, $0.92,95 \%$ CI 0.63 to 1.34$)^{18}$ and this association was confirmed by our study with a borderline significant OR of 0.75 (95\% CI 0.56 to 1.02). There was no correlation between Necessity and Concern (table 2) in our study population and this was supported by a recent Swedish study conducted in 578 stroke patients. ${ }^{19}$ They reported a Spearman's correlation coefficient of $0.075 \quad(p=0.08)$. Compared with patients with high necessity and low concerns, patients with high necessity and high concerns, low necessity and high concerns, and low necessity and low concerns were all associated with non-adherence and this was supported by studies conducted in patients with inflammatory bowel disease and renal dysfunction. ${ }^{20} 21$ High perceived sensitivity to medicines was reported to be associated with non-adherence and higher medical care utilisation, increased symptom reporting and greater information-seeking about medication. ${ }^{1822}$ Our study also showed that PSM was associated with non-adherence.

Our study has some strengths. First, this was the first large BMQ study conducted in China. Second, we choose two large teaching hospitals for this study and collected data continually until the target numbers were reached for each condition. Therefore, our study population was more likely to represent the disease population from each region as large teaching hospitals provide healthcare service to majority of Chinese patients. However, we acknowledge that some patients with transient ischaemic attacks (TIAs), a subtype of stroke may not be 
Table 3 Correlation matrix between Beliefs about Medicines Questionnaire (BMQ)-Specific, BMQ-General and perceived sensitivity to medicines (PSM)

\begin{tabular}{|c|c|c|c|c|c|c|}
\hline & Necessity & Concerns & Overuse & Harm & Benefit & PSM \\
\hline \multicolumn{7}{|l|}{ Overall } \\
\hline Necessity & 1.00 & & & & & \\
\hline Concern & $0.02(0.45)$ & 1.00 & & & & \\
\hline Overuse & $-0.01(0.87)$ & $0.39(<0.01)$ & 1.00 & & & \\
\hline Harm & $-0.05(0.16)$ & $0.49(<0.01)$ & $0.45(<0.01)$ & 1.00 & & \\
\hline Benefit & $0.31(<0.01)$ & $-0.09(0.01)$ & $0.11(<0.01)$ & $0.04(0.21)$ & 1.00 & \\
\hline PSM & $0.04(0.22)$ & $0.45(<0.01)$ & $0.39(<0.01)$ & $0.38<0.01)$ & $0.05(0.14)$ & 1.00 \\
\hline \multicolumn{7}{|l|}{ Stroke } \\
\hline Necessity & 1.00 & & & & & \\
\hline Concern & $0.04(0.43)$ & 1.00 & & & & \\
\hline Overuse & $0.06(0.31)$ & $0.50(<0.01)$ & 1.00 & & & \\
\hline Harm & $-0.06(0.27)$ & $0.66(<0.01)$ & $0.57(<0.01)$ & 1.00 & & \\
\hline Benefit & $0.36(<0.01)$ & $0.01(0.88)$ & $0.24(<0.01)$ & $0.13(0.12)$ & 1.00 & \\
\hline PSM & $0.23(<0.01)$ & $0.52(<0.01)$ & $0.51(<0.01)$ & $0.50(<0.01)$ & $0.22(<0.01)$ & 1.00 \\
\hline \multicolumn{7}{|c|}{ Diabetes mellitus } \\
\hline Necessity & 1.00 & & & & & \\
\hline Concern & $0.02(0.59)$ & 1.00 & & & & \\
\hline Overuse & $-0.05(0.40)$ & $0.26(<0.01)$ & 1.00 & & & \\
\hline Harm & $-0.08(0.20)$ & $0.21(<0.01)$ & $0.44(<0.01)$ & 1.00 & & \\
\hline Benefit & $0.19(<0.01)$ & $-0.07(0.26)$ & $0.18(<0.01)$ & $0.08(0.19)$ & 1.00 & \\
\hline PSM & $-0.08(0.20)$ & $0.36(<0.01)$ & $0.38(<0.01)$ & $0.44(<0.01)$ & $0.07(0.23)$ & 1.00 \\
\hline \multicolumn{7}{|l|}{ RA } \\
\hline Necessity & 1.00 & & & & & \\
\hline Concerns & $0.01(0.91)$ & 1.00 & & & & \\
\hline Overuse & $-0.08(0.13)$ & $0.41(<0.01)$ & 1.00 & & & \\
\hline Harm & $0.02(0.72)$ & $0.42(<0.01)$ & $0.30(<0.01)$ & 1.00 & & \\
\hline Benefit & $0.30(<0.01)$ & $-0.21(<0.01)$ & $-0.20(<0.01)$ & $-0.15(0.21)$ & 1.00 & \\
\hline PSM & $-0.04(0.52)$ & $0.54(<0.01)$ & $0.29(<0.01)$ & $0.27(<0.01)$ & $-0.13(0.02)$ & 1.00 \\
\hline
\end{tabular}

admitted to hospital. Therefore, our stroke inpatients may under-represent the TIA patients. Thirdly, China has higher prevalence rates of stoke and diabetes mellitus than Western Europe and stroke is also the leading cause

Table 4 ORs of medicine non-adherence

\begin{tabular}{lll}
\hline & $\begin{array}{l}\text { Unadjusted } \\
\text { OR, 95\% Cl }\end{array}$ & $\begin{array}{l}\text { Adjusted } \\
\text { OR, 95\% } \mathbf{~ C l ~}\end{array}$ \\
\hline Specific-Necessity & $0.90,0.67$ to 1.21 & $0.93,0.68$ to 1.27 \\
\hline Specific-Concerns & $1.27,1.02$ to 1.58 & $1.35,1.07$ to 1.71 \\
\hline General-Overuse & $1.32,1.04$ to 1.69 & $1.15,0.88$ to 1.50 \\
\hline General-Harm & $1.09,0.87$ to 1.38 & $1.12,0.89$ to 1.43 \\
\hline General-Benefit & $0.84,0.63$ to 1.12 & $0.75,0.56$ to 1.02 \\
\hline PSM & $1.72,1.41$ to 2.09 & $1.44,1.16$ to 1.85 \\
\hline
\end{tabular}

${ }^{*}$ Adjusted for age, gender, education, occupation, duration of the disease and different diseases.

PSM, Perceived Sensitivity to Medicines scale. of death in China. In light of the current deteriorated relationship between health professional and patients (ie, patients do not trust their doctors and other health professionals, doctors, nurses are often abused verbally and physically by patients), ${ }^{11} 2324$ more studies are needed from the patients' perspective. More consideration from the patient perspective should be taken into account in terms of chronic disease management and the relationship between patients and health professionals. However, our study was an observational study. Therefore, the results may be confounded by unmeasured factors such as comorbidity. Self-reported adherence may not be the best measurement for medicine adherence as patients may, for example, underestimate how often they forget their treatment. However, a strong correlation was found for medicine adherence measured by self-reported questionnaire, electronic prescriptions and serum biomarkers in a recent intervention study suggesting that self-report is a reliable measure..$^{25}$ Also, our study found relatively low 
Table 5 ORs of medication non-adherence by disease groups

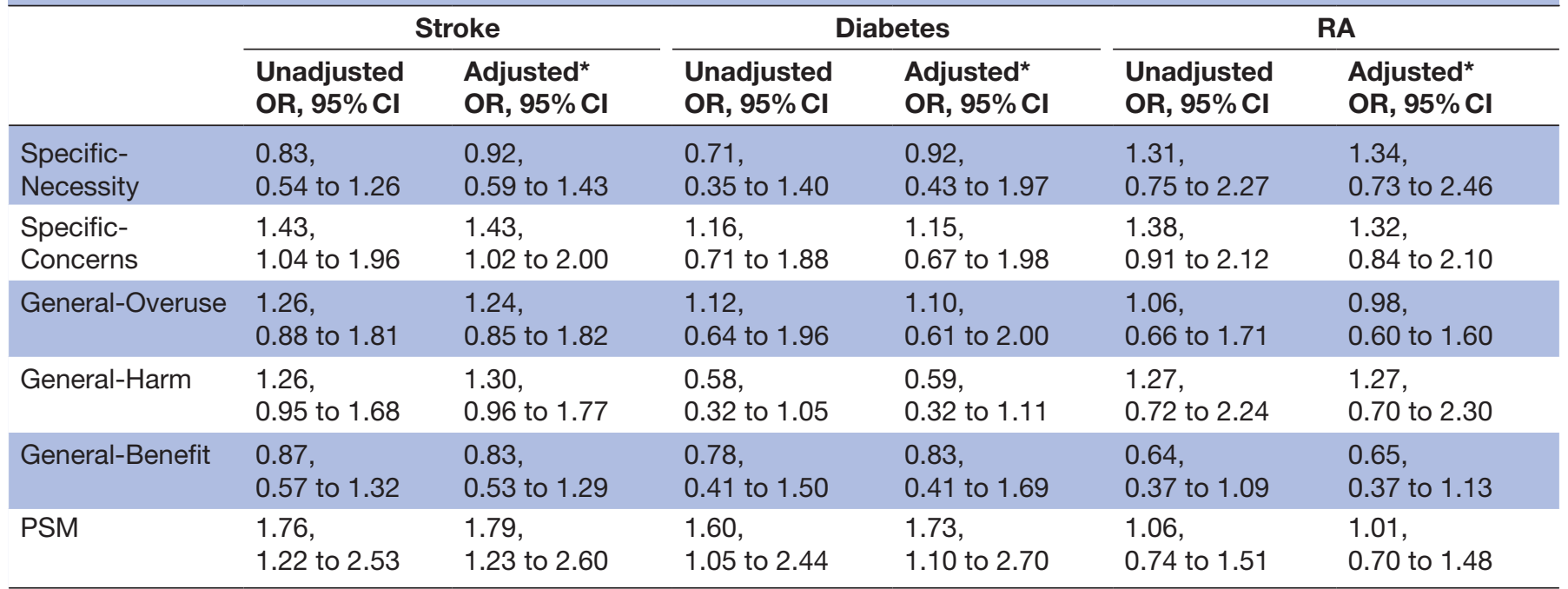

*Adjusted for age, gender, education, occupation, duration of the disease.

PSM, Perceived Sensitivity to Medicines scale; RA, rheumatoid arthritis.

reliability of the BMQ general scales and further investigation and refinement might be needed of these Chinese translations. The difference in non-adherence between stroke and other conditions needs to be explored in future studies as disease itself may play an important role in medicine adherence.

In conclusion, we found that the BMQ is a useful tool to identify psychological factors that are linked to non-adherence in patients with stroke, diabetes and RA. Future studies should use the BMQ to screen patients to identify those who are at high risk of non-adherence and map their treatment plan accordingly.

\section{Author affiliations}

${ }^{1}$ Department of Practice and Policy, University College London School of Pharmacy, London, UK

${ }^{2}$ Department of Pharmacy \& Pharmacology, University of Bath, Bath, UK

${ }^{3}$ Department of Rheumatology and Immunology, Anhui Provincial Hospital, Anhui Medical University, Hefei, China

${ }^{4}$ Department of Neurology, The Second Hospital, Tianjin Medical University, Tianjin, China

${ }^{5}$ Department of Endocrinology, Anhui Provincial Hospital, Anhui Medical University, Hefei, China

${ }^{6}$ Centre for Health and Social Care Improvement, Faculty of Education Health and Wellbeing, University of Wolverhampton, Wolverhampton, UK

${ }^{7}$ Centre for Health, Wellbeing and Behaviour Change, Faculty of Education and Sport, University of Bedfordshire, Bedford, UK

Contributors LW, RC and RH were involved in the conception and design and interpretation of data. LW did the statistical analysis and wrote the first draft of the article. SC, RC and BN contributed to data analysis. LXM, LX and LSM collected data. SC, AC, LXM, LX and LSM were involved in interpretation of data. All authors were responsible for re-drafting of the article and approved the final version.

Funding This study was funded by the Research Innovation Fund, UCL School of Pharmacy. The funder had no role in the design, conduct or data interpretation of the study. Professor Rob Horne was supported by the National Institute for Health Research (NIHR) Collaboration for Leadership in Applied Health Research and Care (CLAHRC) North Thames at Bart's Health NHS Trust.

Competing interests RH declares: (1) Speaker engagements with honoraria and consultancy payments from the pharmaceutical companies; (2) Founder and shareholder of a UCL-Business, university spin-out company (Spoonful of Sugar) providing consultancy on medication-related behaviours to healthcare policy makers, providers and industry; and (3) Originator of BMQ.

Patient consent Study was conducted outside of the UK. Study information was given to patients and verbal consent was obtained before they started to fill in the questionnaire.

Ethics approval Local Hospital Research Committee.

Provenance and peer review Not commissioned; externally peer reviewed.

Data sharing statement No additional data available.

Open Access This is an Open Access article distributed in accordance with the Creative Commons Attribution Non Commercial (CC BY-NC 4.0) license, which permits others to distribute, remix, adapt, build upon this work non-commercially, and license their derivative works on different terms, provided the original work is properly cited and the use is non-commercial. See: http://creativecommons.org/ licenses/by-nc/4.0/

(c) Article author(s) (or their employer(s) unless otherwise stated in the text of the article) 2017. All rights reserved. No commercial use is permitted unless otherwise expressly granted.

\section{REFERENCES}

1. Medicines adherence: involving patients in decisions about prescribed medicines and supporting adherence. https://www.nice. org.uk/guidance/cg76/chapter/introduction (accessed on 30 January 2017).

2. Liu L, Wang D, Wong KS, et al. Stroke and stroke care in China: huge burden, significant workload, and a national priority. Stroke; a journal of cerebral circulation.2011;42:3651-4.

3. Feigin VL, Forouzanfar MH, Krishnamurthi R, et al. Global and regional burden of stroke during 1990-2010: findings from the Global Burden of Disease Study 2010. Lancet 2014;383:245-55.

4. Li R, Sun J, Ren LM, et al. Epidemiology of eight common rheumatic diseases in China: a large-scale cross-sectional survey in Beijing. Rheumatology 2012;51:721-9.

5. China Total population2015 http://www.who.int/countries/chn/en/ (accessed on 25 May 2017).

6. Wu X, Zhu B, Fu L, et al. Prevalence, incidence, and mortality of stroke in the chinese island populations: a systematic review. PLoS One 2013;8:e78629 eCollection 2013.

7. Will JC, Zhang Z, Ritchey MD, et al. Medication adherence and incident preventable hospitalizations for hypertension. Am J Prev Med 2016;50:489-99.

8. Shore S, Carey EP, Turakhia MP, et al. Adherence to dabigatran therapy and longitudinal patient outcomes: insights from the veterans health administration. Am Heart J 2014;167:810-7. 
9. Korhonen MJ, Ruokoniemi P, llomäki J, et al. Adherence to statin therapy and the incidence of ischemic stroke in patients with diabetes. Pharmacoepidemiol Drug Saf 2016;25:161-9.

10. Horne R, Chapman SC, Parham R, et al. Understanding patients adherence-related beliefs about medicines prescribed for longterm conditions: a meta-analytic review of the Necessity-Concerns Framework. PLoS One 2013;8:e80633.

11. Tucker JD, Cheng Y, Wong B, et al. Patient-physician trust project team. Patient-physician mistrust and violence against physicians in Guangdong Province, China: a qualitative study. BMJ Open 2015;5:e008221.

12. Chan CSC, Cheng Y, Cong $Y$, et al. Patient-physician trust in China: a workshop summary. The Lancet 2016;388(Suppl 1):S72.

13. National health and family planning cdommission of the PRC. http:// en.nhfpc.gov.cn/ (last access 30 Jan 2017).

14. Horne R, Weinman J. Patients' beliefs about prescribed medicines and their role in adherence to treatment in chronic physical illness. $J$ Psychosom Res 1999;47:555-67.

15. Horne R. The nature determinants and effects of medication beliefs in chronic illnessPhD thesis university of London. 1997.

16. Bland JM, Altman DG. Statistics notes: Cronbach's alpha. BMJ 1997;314:572 https://doi.org/re10.1136/bmj.314.7080.572.

17. Wei L, Wang J, Thompson P, et al. Adherence to statin treatment and readmission of patients after myocardial infarction: a six year follow up study. Heart 2002;88:229-33.
18. Chapman SC, Horne R, Chater A, et al. Patients' perspectives on antiepileptic medication: relationships between beliefs about medicines and adherence among patients with epilepsy in UK primary care. Epilepsy Behav 2014;31:312-20.

19. Sjölander M, Eriksson M, Glader EL. The association between patients' beliefs about medicines and adherence to drug treatment after stroke: a cross-sectional questionnaire survey. BMJ Open 2013;3:e003551.

20. Horne R, Parham R, Driscoll R, et al. Patients' attitudes to medicines and adherence to maintenance treatment in inflammatory bowel disease. Inflamm Bowel Dis 2009;15:837-44.

21. Chater AM, Parham R, Riley S, et al. Profiling patient attitudes to phosphate binding medication: a route to personalising treatment and adherence support. Psychol Health 2014;29:1407-20.

22. Faasse K, Grey A, Horne R, et al. High perceived sensitivity to medicines is associated with higher medical care utilisation, increased symptom reporting and greater information-seeking about medication. Pharmacoepidemiol Drug Saf 2015;24:592-9.

23. Zg L, Leng MX. Doctor-patient relationship research summary. Chinese Hospital Management 2009;29:40-3.

24. Doctors face verbal abuse or violence. http://www.cmdae.org/en/ index.php (Accessed 30 January 2017).

25. Lyons I, Barber N, Raynor DK, et al. The Medicines Advice Service Evaluation (MASE): a randomised controlled trial of a pharmacistled telephone based intervention designed to improve medication adherence. BMJ Qual Saf 2016;25:759-69. 